\title{
Trauma craneoencefálico. Revisión de la literatura
}

\author{
José D. Charry MD MS(c) ${ }^{1 a}$, Juan F. Cáceres MD², Andrea C. Salazar MD², Lisseth P. López MD², Juan P. Solano MD. ${ }^{3}$ \\ 1 Médico Investigador, Fundación Universitaria Navarra, UNINAVARRA, Neiva, Colombia. \\ 2 Médico Investigador, Clínica Mediláser S.A, Neiva, Colombia. \\ 3 Neurocirujano, Clínica Mediláser S.A. Neiva, Colombia. \\ * Universidad de Jaén, España.
}

Rev. Chil. Neurocirugía 43: 177-182, 2017

\begin{abstract}
Resumen
El trauma craneoencefálico (TCE) es una patología médico-quirúrgica caracterizada por una alteración cerebral secundaria a una lesión traumática en la cabeza generando un daño estructural del contenido de ésta, incluyendo el tejido cerebral y sus vasos sanguíneos. La incidencia es de 200 personas por cada 100.000 habitantes, la etiología más frecuente son los accidentes de tránsito $(70 \%)$, seguidos de hechos violentos y/o caídas desde su propia altura dependiendo del área geográfica en el que se encuentre. En esta revisión, se explicarán las lesiones primarias, secundarias, terciarias, el deterioro retardado, los tipos de hipertensión endocraneana y las lesiones específicas como fisiopatología del TCE, así como la evaluación clínica e imagenológica de las lesiones y su adecuado tratamiento.
\end{abstract}

Palabras clave: Trauma craneoencefálico, epidemiología, fisiopatología, tratamiento.

\begin{abstract}
Cranioencephalic trauma (TBI) is a medical-surgical pathology characterized by a cerebral alteration secondary to a traumatic injury to the head causing structural damage to this contents, including the brain tissue and its blood vessels. The incidence is 200 people per 100,000 inhabitants, the most frequent etiology in traffic accidents (70\%), followed by violent events and / or fall from their own height depending on the geographical area in which they are. Primary, secondary, tertiary injuries, delayed deterioration, types of endocranial hypertension and specific lesions such as TBI pathophysiology, as well as clinical and imagenologyc evaluation of lesions and their appropriate treatment are explained in this review.
\end{abstract}

Key words: Traumatic Brain Injury, epidemiology, pathophysiology, treatment.

\section{Introducción}

El trauma craneoencefálico (TCE) se define como una patología médicoquirúrgica caracterizada por una alteración cerebral secundaria a una lesión traumática en la cabeza con la presencia de al menos uno de los siguientes elementos: alteración de la consciencia y/o amnesia debido al trauma; cambios neurológicos o neurofisiológicos, o diagnóstico de fractura de cráneo o lesiones intracraneanas atribuibles al trauma ${ }^{1}$, producto de la liberación de una fuerza externa ya sea en forma de energía mecánica, química, térmica, eléctrica, radiante o una combinación de éstas, resulta en un daño estructural del contenido de ésta, incluyendo el tejido cerebral y los vasos sanguíneos que irrigan este tejido ${ }^{2,3}$. También se define como la ocurrencia de muerte resultante del trauma que incluya los diagnósticos de lesión de la cabeza y /o injuria cerebral traumática entre las causas que produjeron la muerte ${ }^{1}$.

\section{Epidemiología}

Se estima que, la incidencia de TCE a nivel mundial es alrededor de 200 personas por cada 100.000 habitantes, que por cada 250-300 TCE leves hay 15-20 moderados y 10-15 graves. La relación es 2:3 afectando más a los hombres, con una edad de máximo riesgo situada entre los 15 y los 30 años $^{4}$, por lo que se considera un problema de salud pública. La etiología más frecuente son los accidentes 
de tránsito $(70 \%)^{5}$, seguidos de hechos violentos y/o caídas desde su propia altura dependiendo del área geográfica en el que se encuentre. La tasa global de mortalidad por trauma es de 19 por 100.000 habitantes; en América Latina de 75.5 por 100.000 habitantes, y en el Colombia, de 125 por 100.000 habitantes.

En España, la incidencia no varía y de estos casos, el $70 \%$ tienen una buena recuperación, el $9 \%$ fallecen antes de llegar al hospital, el 6\% lo hacen durante su estancia hospitalaria y el $15 \%$ quedan funcionalmente incapacitados en diferentes grados (moderados, graves o vegetativos) ${ }^{4}$. En Argentina, la incidencia es de 322 por 100.000 habitantes, de los cuales un $93 \%$ corresponde a TCE leves; $4 \%$ a TCE moderados y $3 \%$ a TCE grave ${ }^{6}$.

Un estudio publicado por la universidad de Salamandra en el $2014^{5}$, reportó que en Cali (Colombia) el registro de muertes anuales por TCE fue cercano a 1.000 , donde la mitad de estas estuvieron asociadas con automotores y alcohol, afectando en un $60 \%$ a hombres jóvenes ${ }^{5}$, y que el $72 \%$ de las consultas por TCE en el Hospital Universitario del Valle fueron producto de accidentes de tránsito y que de ese porcentaje, el $16 \%$ asociado a TCE grave ${ }^{5}$, no muy lejos de las cifras mundiales (11-16 casos por cada 100.000 habitantes) ${ }^{1}$. Además, datos del Instituto Colombiano de Medicina Legal y Ciencias Forenses de 2014 (FORENSIS) $^{7}$ revela estadísticas donde demuestran que el TCE es causado principalmente por eventos violentos (homicidio por TCE 2.516 casos $(23,29 \%)^{7}$, violencia interpersonal con TCE 2742 casos $(2,55 \%)^{7}$, Violencia infantil con TCE un total de 105 casos $(1,56 \%)^{7}$, violencia en pareja con TCE 445 casos $(1,12 \%)$, y violencia contra el adulto mayor con TCE reportó un total 34 casos $(3,27 \%)^{7}$.

Otro estudio colombiano (EPINEURO) ${ }^{8}$ del año 2003 revela que la prevalencia de secuelas de traumatismo craneoencefálico es superior a la publicada para Canadá, Estados Unidos y Escocia $^{8}$, lo cual puede estar asociado a problemas socioculturales que aquejan al país como es el caso de la violencia, y se ve reflejado entre los hombres $(7,5 \text { por } 1.000 \text { habitantes })^{8}$, los cuales se asocian más a hechos violentos, especialmente entre los de más de 65 años (23,6 por 1.000 habitantes). Las mayores frecuencias se observaron en la costa caribe y en la región noroccidental (zona concurrida por el narcotráfico y paramilitares). EI TCE constituye entre $33 \%$ y $50 \%$ de las causas de muerte ${ }^{9}$. De estos, $50 \%$ fallecen en minutos, $30 \%$ en menos de 2 horas y $20 \%$ fallecen después. Del total de las muertes por accidente de tránsito, $60 \%$ es causado primariamente por TCE. ${ }^{9}$

\section{Fisiopatología}

\section{Lesión primaria}

Es el daño directo tras el impacto debido a su efecto biomecánico o por aceleración-desaceleración. En relación con el mecanismo y la energía transferida, se produce lesión celular, desgarro y retracción axonal y alteraciones vasculares. Depende de la magnitud de las fuerzas generadas, su dirección y lugar de impacto ${ }^{10}$. Hay lesiones focales como la contusión cerebral, en relación con fuerzas inerciales directamente dirigidas al cerebro y lesiones difusas, como la lesión axonal difusa, en relación con fuerzas de estiramiento, cizallamiento y rotación ${ }^{9}$.

\section{Lesión secundaria}

Sucede por una serie de procesos metabólicos, moleculares, inflamatorios e incluso vasculares, iniciados con el traumatismo, activando cascadas que incrementan la liberación de aminoácidos excitotóxicos (glutamato) que activan receptores MNDA/AMPA los cuales alteran la permeabilidad de membrana (aumentando el agua intracelular, liberan potasio al exterior y permiten la entrada masiva de calcio en la célula), estimulando la producción de proteinasas, lipasas y endonucleasas que desencadenan la muerte celular inmediata por necrosis o por apoptosis celular $^{10}$. En el TCE grave se produce activación del estrés oxidativo, aumentando los radicales libres de oxígeno y N2, generando daño mitocondrial y del ADN. Estas lesiones son agravadas por daños intracraneales (lesión masa, hipertensión intracraneal, convulsiones, etc.) como extracraneales (hipoxia, hipotensión, hipoventilación, hipovolemia, coagulopatía, hipertermia, etc.) ${ }^{9}$.

\section{Lesión terciaria}

Es la manifestación tardía de los daños progresivos o no ocasionados por la lesión primaria y secundaria con necrosis, apoptosis y/o anoikis (muerte celular programada por desconexión, que produce eventos de neurodegeneración y encefalomalasia, entre otros..$^{9,11-17}$

\section{Deterioro retardado}

Corresponde al $15 \%$ de los pacientes con TCE que no manifiestan síntomas o signos de lesión cerebral, pero en el transcurso de minutos $u$ horas presentan un deterioro neurológico por lesiones que pueden llegar a ser fatales ${ }^{9}$. Por lo anterior, todo paciente con TCE debe ser vigilado durante 24 horas 0 hasta la resolución de su síndrome de base, además se debe tomar un TAC de cráneo en las primeras 6 horas, el cual se repite antes de dar egreso o si el paciente presenta síntomas o signos neurológicos ${ }^{9,16,18}$.

\section{Hipertensión endocraneana (HTEC) de tipo difusa}

Ocurre por el aumento agudo y difuso de todos o alguno de los contenidos intracraneanos, que causará un aumento de la PIC de tipo difuso. Los principales síntomas son cefalea persistente, vértigo y diplopía. Algunos de los signos son: Deterioro de la conciencia, papiledema y VI par, reflejo de Cushing, tríada de Cushing, relacionado con herniación cerebelo-tonsilar y compresión del bulbo ${ }^{11-17}$.

\section{Hipertensión endocraneana focal}

Aumento de la presión a nivel focal con efecto de masa sobre las estructuras vecinas, que en ocasiones requiere manejo quirúrgico. Los síntomas son iguales a la HTEC difusa. Los signos son: III par craneal, convulsión focal, hemiparesia contralateral a la lesión, herniación cerebral14,18,19, 20-27 cingular o subfalcinam, herniación uncal, trancraneana, tonsilar o de amigdalas cerebelosas, herniación central transtentorial, transtentorial inversa, hipotensión y el síndrome del segundo impacto ${ }^{11-17}$. Algunos ejemplos son las lesiones específicas que se hablaran en el siguiente párrafo.

\section{Lesiones específicas}

\section{Hematoma epidural}

Se observa a través del TAC como una lesión hiperdensa en forma biconvexa o «en forma de lente», se comporta como 
HTEC focal, que requerirá craneotomía y drenaje quirúrgico dependiendo su tamaño. Corresponde al $1-6,5 \%$ de todos los TCE. La causa más frecuente es la ruptura arterial (85\%), La mortalidad es entre $5 \%$ y $10 \%$ con tratamiento quirúrgico a tiempo ${ }^{9}$.

\section{Hematoma subdural}

Originado por una lesión en plexos venosos subdurales y venas puente que van hacia los senos venosos durales. Se observa en la TAC como una lesión hiperdensa, isodensa o hipodensa (según el tiempo de evolución) en forma de semiluna. Corresponde al $5,6 \%$ del TCE en general y $30 \%$ de todos los TCE Severos. La mortalidad es de $60 \%$ con tratamiento, subiendo a $90 \%$ en los pacientes operados en coma (Glasgow < 9). Se clasifica según el tiempo de evolución en: Agudos: menor de 24 horas de evolución $\left(\mathrm{M}^{*}\right.$ entre $50 \%$ y $90 \%$ ); Subagudos: mayor de 24 horas y menor de 7 días, (M: 25\%) y Crónicos: mayor de 7 días, (M: $50 \%)^{9}$.

\section{Contusión cerebral}

Corresponde al $9 \%$ de los TCE, por mecanismos de y contragolpe. Pueden ser hemorrágico y no hemorrágicos, se evidencian en la TAC como una lesión redondeada o elíptica, con edema perilesional y efecto de masa. Su comportamiento es amenazante entre el día 4 y 7 debido al efecto de masa por la contusión y el edema con picos. Es necesario no confundir los términos con de Contusión cerebral con Concusión cerebral (Lesión cerebral difusa, con desregulación eléctrica neuronal) ni Conmoción cerebral (pérdida del conociendo en un paciente con TCE, independiente de la lesión que presente) ${ }^{9}$.

\section{Evaluación}

\section{Exploración general}

Se debe descartar las posibles lesiones que puedan generar: trastornos respiratorios (contusión pulmonar, fracturas costales múltiples, broncoaspirado de sangre o vómito), trastornos cardiovasculares (hipovolemia por rotura hepatoesplénica, taponamiento cardíaco, contusión miocárdica, shock medular), debido a su relación con el daño cerebral secundario ${ }^{10}$ el cual oscila entre un $25 \%$ y un $88 \%$ de los casos $^{18}$; también

${ }^{\star} \mathrm{M}$ : Tasa de muerte. es necesario prevenir una lesión medular que pueda ser ocasionado por una inadecuada movilización en pacientes politraumatizados o con trauma en columna. Asimismo, descartar disección carotídea que pueden ocasionar infartos cerebrales; para ello, hay que estar atentos a hematomas en la unión cervicotorácica o isquemia hemisférica precoz en ausencia de focos contusivos en pacientes con trauma cervical ${ }^{10}$.

\section{Examen neurológico}

Se utiliza la Escala de Coma de Glasgow la cual evalúa 3 parámetros: apertura palpebral, respuesta verbal y respuesta motora, a los que se da una puntuación según el tipo de respuesta y al se establecen 3 categorías: - TCE Leve (Glasgow 13-15) - TCE Moderado (Glasgow 9-12) - TCE Severo (Glasgow 3-8) las cuales están correlacionados con la severidad ${ }^{2}$, como se muestra en la siguiente Tabla 1:

Valoración del estado de las pupilas El tamaño y asimetría pupilares demuestran la gravedad de la lesión cerebral y su localización. Las alteraciones pupilares son miosis y midriasis y dependen del compromiso promovido so- bre el arco relejo fotomotor en alguno de sus eslabones.

Miosis (constricción de 1-3 mm) Sucede al inicio de la herniación centroencefálica, por compromiso de los axones simpáticos originados en el hipotálamo, haciendo predominar la acción parasimpática, transmitida por el III par (tono pupiloconstrictor de base en el músculo ciliar del ojo). Se observa unilateral en la herniación transtentorial con compresión mesencefálica; intensa en las lesiones pontomesencefálicas dorsales (núcleo rojo) que interrumpen la señal descendente que termina en el ganglio estrellado, antes de ascender por el plexo nervioso pericarotídeo ${ }^{10}$.

\section{Midriasis (dilatación $\geq 6 \mathrm{~mm}$ )}

Se da por lesión del III par, seguido de la disfunción de sus axones parasimpáticos, lo que detiene las señales eferentes para la constricción pupilar ${ }^{10}$. Ocurre principalmente por herniación del uncus, por gradiente de presión transtentorial, el cual comprimirá al III par ipsolateral y al pedúnculo mesencefálico ${ }^{10}$. Se observa pupilas medias o débilmente dilatadas $(6 \mathrm{~mm})$, redondas o raramente discóricas, con un relejo

\begin{tabular}{|c|c|c|}
\hline $\begin{array}{l}\text { Respuesta ocularo } \\
\text { apertura palpebral }\end{array}$ & Respuesta verbal & Respuesta motora \\
\hline Sin apertura ocular (1) & Sin respuesta verbal (1) & Sin respuesta motora (1) \\
\hline Al estímulo Doloroso (2) & $\begin{array}{l}\text { Sonidos incomprensibles } \\
\text { o guturales (2) }\end{array}$ & $\begin{array}{l}\text { Respuesta anormal en } \\
\text { extensión o descerebra- } \\
\text { ción (2) }\end{array}$ \\
\hline Al estímulo Auditivo (3) & $\begin{array}{l}\text { Palabras fuera de contex- } \\
\text { to (respuestas inapropia- } \\
\text { das) (3) }\end{array}$ & $\begin{array}{l}\text { Respuesta anormal en } \\
\text { flexión o decorticación (3) }\end{array}$ \\
\hline \multirow[t]{3}{*}{ Espontánea (4) } & $\begin{array}{l}\text { Desorientación en alguna } \\
\text { de las } 3 \text { esferas (confuso) } \\
\text { (4) }\end{array}$ & $\begin{array}{l}\text { Retira ante estímulos no- } \\
\text { ciceptivos o dolorosos (4) }\end{array}$ \\
\hline & Orientado en 3 esferas (5) & $\begin{array}{l}\text { Localiza estímulos noci- } \\
\text { ceptivos o dolorosos (5) }\end{array}$ \\
\hline & & $\begin{array}{l}\text { Obedece órdenes o rea- } \\
\text { liza movimientos espon- } \\
\text { táneos (6) }\end{array}$ \\
\hline
\end{tabular}

Para una puntuación de 3 cabe esperar una tasa BR del $4,1 \%$ y del $85 \%$ de tasa de muerte + estado vegetativo postraumático (M+EVP), para una puntuación de 4, las tasas son $6,3 \%$ (BR) y $67 \%$ (M+EVP); para 5 puntos, $12,2 \%$ (BR) y $47,5 \%$ (M+EVP); para 6 puntos, $29,2 \%$ (BR) y $26,5 \%$ (M+EVP); para 7 puntos, $46,6 \%$ (BR) y $18,6 \%$ (M+EVP); y para 8 puntos, $54,8 \%(B R)$ y $19 \%(M+E V P)^{19,20}$. 
fotomotor débil o ausente en las lesiones mesencefálicas dorsales (lámina cuadrigémina), midriasis paralítica (8-9 $\mathrm{mm}$ ) en las lesiones mesencefálicas ventrales (pedúnculos cerebrales), midriasis bilateral por sección mesencefálica. En ocasiones se aprecia anisocoria por traumatismo orbitario y daño asociado del nervio óptico.

\section{Valoración de los movimientos ocu-} lares extrínsecos

\section{Centros protuberanciales}

Existe un centro pontobulbar simétrico (uno a la derecha y otro a la izquierda), en conexión con las fibras inervatorias del recto interno (III par) y con las del motor ocular lateral (VI par), cuyo estímulo 'obliga' a ambos ojos a dirigirse conjugadamente en una dirección ipsolateral al mismo en rápidos pulsos o sacadas (reflejo oculocefálico).

\section{Centros corticales}

También existen otros núcleos -derecho e izquierdo- de la mirada conjugada en los lóbulos frontales, cuya estimulación induce impulsos tónicos contralaterales en los ojos, manteniéndo- los al frente en condiciones normales.

\section{Neuroimagen}

La Tomografía Computarizada (TC) es la imagen de elección para el diagnóstico, pronóstico, control evolutivo de las lesiones iniciales del TCE y de la respuesta a la terapia ${ }^{20}$. Es necesario considerar si el paciente está subreactivo desde el impacto (lesión difusa) y si la lesión quirúrgica (hematoma, contusión o mixta), tiene un volumen mayor o menor de $25 \mathrm{ml}$ (lesión focal). El volumen de la lesión se calcula (en mililitros) multiplicando los tres diámetros de la lesión (en centímetros) y dividiéndolos por 2 si su morfología tiende a ser esférica, o por 3 si su morfología se asemeja a un elipsoide ${ }^{20}$. Con el fin de clasificar las lesiones por TCE en TAC, se creó la escala de Marshall (Tabla 2).

\section{Monitorización de la presión intra- craneal (PIC)}

Permite prevenir el deterioro neurológico y controlar la efectividad de las terapias empleadas. Los sistemas más utilizados son los transductores acoplados a fluidos (catéter intraventricular) y los sensores intraparenquimatosos ${ }^{10}$. Las guías recomiendan monitorizar a todos los pacientes con TCE grave con TAC patológico, y a los pacientes con TCE grave y TAC normal tengan dos o más de las siguientes características: edad $\geq 40$ años, respuestas motoras francamente anormales $(M \leq 4)$ y tensión arterial $\leq 90 \mathrm{~mm} \mathrm{Hg}$ en algún momento de su evolución ${ }^{22}$.

\section{Tratamiento}

\section{En el lugar del accidente}

Es necesario prevenir el daño secundario, para ello es necesario: detener hemorragias, evitar la broncoaspiración, la hipotensión, disminuir el dolor, y aportar oxígeno cuando se sospeche daño sistémico ${ }^{10}$.

\section{En el transporte}

A menos de que el paciente requiera reanimación cardiopulmonar (RCP) en el sitio del accidente, se debe trasladar en posición neutra supina, con collar cervical, controlándose perfectamente

Tabla 2.

Clasificación tomográfica de Marshall o del Traumatic Coma Data Bank ${ }^{21}$

\begin{tabular}{|c|c|c|c|c|c|}
\hline Categoría & Definición & Cisternas & DLM $^{4}$ & $\begin{array}{l}\text { Lesión tipo } \\
\text { masa }\end{array}$ & Mortalidad \\
\hline $\begin{array}{l}\text { Lesión Di- } \\
\text { fusa I }\end{array}$ & Patología intracraneal no visible & Normales & Ninguna & Ninguna & $9,6 \%$ \\
\hline $\begin{array}{l}\text { Lesión Difu- } \\
\text { sa II }\end{array}$ & $\begin{array}{l}\text { Cisternas presentes con desviación de línea media } \\
\text { de } 0-5 \mathrm{~mm} \text { y/o: presencia de lesiones densas de } \\
\text { densidad alta o mixta no mayor a } 25 \mathrm{cc} \text {. Puede } \\
\text { incluir fragmentos óseos o cuerpos extraños }\end{array}$ & Presentes & $0-5 \mathrm{~mm}$ & $\begin{array}{l}\text { Ninguna }> \\
25 \text { cc }\end{array}$ & $13,5 \%$ \\
\hline $\begin{array}{l}\text { Lesión Difu- } \\
\text { sa III (Ede- } \\
\text { ma) }\end{array}$ & $\begin{array}{l}\text { Cisternas comprimidas o ausentes con desviación } \\
\text { de línea media entre 0-5 mm; No hay lesiones de } \\
\text { alta o mixta densidad mayores de } 25 \mathrm{cc}\end{array}$ & $\begin{array}{l}\text { Comprimidas o } \\
\text { Ausentes }\end{array}$ & $0-5 \mathrm{~mm}$ & $\begin{array}{l}\text { Ninguna }> \\
25 \mathrm{cc}\end{array}$ & $34 \%$ \\
\hline $\begin{array}{l}\text { Lesión Difu- } \\
\text { sa IV }\end{array}$ & $\begin{array}{l}\text { Desviación de línea media }>5 \mathrm{~mm} \text {. Lesiones de } \\
\text { densidad alta o mixta no mayores a } 25 \mathrm{cc}\end{array}$ & $\begin{array}{l}\text { Comprimidas o } \\
\text { Ausentes }\end{array}$ & $>5 \mathrm{~mm}$ & $\begin{array}{l}\text { Ninguna }> \\
25 \mathrm{cc}\end{array}$ & $56,2 \%$ \\
\hline $\begin{array}{l}\text { Masa Eva- } \\
\text { cuada }\end{array}$ & Cualquier lesión quirúrgicamente evacuada & & & $\begin{array}{l}\text { Ninguna }> \\
25 \mathrm{cc}\end{array}$ & $38,8 \%$ \\
\hline $\begin{array}{l}\text { Lesión Tipo } \\
\text { Masa No } \\
\text { Evacuada }\end{array}$ & $\begin{array}{l}\text { Lesión de densidad alta o mixta mayor a } 25 \text { cc no } \\
\text { evacuada }\end{array}$ & & & $\begin{array}{l}\text { Masa }>25 \\
\text { cc }\end{array}$ & $52,8 \%$ \\
\hline \multicolumn{6}{|c|}{$\begin{array}{l}\text { Tabla 2. Clasificación de Marshall para TCE basada en hallazgos tomográficos a la llegada del paciente a urgencias }{ }^{21} \text {. } \\
2 \text { BR: Tasa de buena recuperación postraumática } \\
{ }^{3} \text { EVP: tasa de estado vegetativo postraumático } \\
{ }^{4} \text { DLM: Desviación de la línea media. }\end{array}$} \\
\hline
\end{tabular}


la vía aérea, con el fin de evitar la broncoaspiración ${ }^{10}$.

\section{En la sala de urgencias}

Se evaluará los parámetros sistémicos, neurológicos y radiológicos con el fin de clasificar la gravedad. Si es TCE grave o moderado se deberá realizar una TAC cerebral y luego será dejados para observación y tratamiento. A los pacientes con TCE leve, se les dará egreso si durante 48 horas posterior al trauma no presenta: disminución del nivel de conciencia, cefalea significativa, amnesia peritraumática o focalización neurológica, fractura craneal, sospecha de intoxicación, si no tienen factores de riesgo (edad $\geq 70$ años, anticoagulación, hepatopatía, ACV o TCE previos, vive solo y/o muy distante del centro hospitalario y el nivel intelectual del acudiente es inapropiado), ya que la tasa de morbimortalidad es del $0 \%{ }^{10}$; de lo contrario, se les realizar una TAC cerebral y se oberva por 48 horas, debido a que la morbimortalidad aumenta al $1 \%{ }^{10}$.

\section{En la sala de neurocirugía}

Los pacientes con TCE moderado o leve, sin daño sistémico asociado, se dejan en observación con una TC de control a las $12-24$ horas de su admisión, con el fin de determinar medidas de vigilancia, control y tratamiento ${ }^{10}$.

\section{En la Unidad de Cuidado Intensivo -} UCl

Deben permanecer los pacientes con GCS $\leq 8$ y aquéllos, en otras categorías que se deterioran, debido a que no hay que descartar la posibilidad de cirugía si los parámetros fisiológicos y radiológicos lo indican. Para ello se tendrá en cuenta los siguientes puntos:

\section{Control de la hipoxia cerebral}

Todo paciente con TCE grave o con un GCS > 8 con daño sistémico será sometido a ventilación mecánica mínimo durante 24 horas, vigilando la hiperventilación para evitar la isquemia provocada por la vasoconstricción que promueve la hipocapnia ${ }^{23}$. En la Hemorragia Subaracnoidea postraumática se recomienda usar calcioantagonistas como lo es dihidropiridina de nimodipi$\mathrm{no}^{24}$.

\section{Control de la complianza}

El objetivo inicial es evitar que la complianza craneoespinal se agote, para ello es necesario: Impedir retener fluido intracraneal facilitando el retorno venoso con la cabeza del paciente a $20^{\circ}$ sobre el plano horizontal ${ }^{10}$; evitar atar el tubo orotraqueal alrededor del cuello; y limitar el empleo de la presión espiratoria final positiva a la estricta indicación de cortocircuito pulmonar (mantenerla en el rango de $\left.5-10 \mathrm{~cm} \mathrm{H}_{2} \mathrm{O}\right)^{25}$.

\section{Tratamiento del edema}

Varios estudios no han demostrado ninguna ventaja en el empleo de dexametasona que, por el contrario, contribuye a la aparición de efectos adversos (hemorragia digestiva, hiperglucemia, trombosis cerebral vascular, inmunosupresión). No debe tampoco olvidarse la existencia de dos tipos especiales de edema celular que se asocian con: el síndrome de secreción inadecuada de hormona antidiurética (SIADH) y el síndrome de depleción salina cerebral $(\mathrm{CSW})^{10}$

El tratamiento es controvertido pero básicamente pueden seguirse los siguientes pasos: a) Restricción hídrica (500 a $1.200 \mathrm{ml} / 24 \mathrm{~h}$ de suero salino); b) Cuando, a pesar de este régimen, no se logra normalizar la natremia en 72 horas o hay deterioro neurológico sin correlación radiológica u oximétrica, se puede proceder con una perfusión de $0,5 \mathrm{ml} / \mathrm{Kg} / \mathrm{h}$ de suero salino al $0,9 \%$ (que contiene $154 \mathrm{mEq}$.L-1) si la natremia es mayor de 125 mEq.L-1 o de suero salino al $3 \%$ si la natremia es menor de 125 mEq.L-1.

\section{Control de la hipertensión intracra- neal}

Una vez agotada la complianza, la PIC comienza a ascender. El manejo de ésta puede realizarse en este orden:

\section{Drenaje ventricular de LCR}

Evacuación repetida de 2-3 $\mathrm{ml}$ de LCR a través del catéter de la ventriculostomía sin exceder los 4-5 días debido a que se asocia a una elevada tasa de infección.

\section{Deshidratación}

Usar manitol al 20\% (dosis rápidas de $0,25-1 \mathrm{mg} / \mathrm{Kg}$ ), tantas veces como resulten eficaces, teniendo en cuenta que: la presión venosa central no debe bajar de $5 \mathrm{~cm}$ de $\mathrm{H}_{2} \mathrm{O}$ y la osmolaridad plasmática debe mantenerse en 310320 mOsm.L-1; el empleo por más de 7 días o los estados hiperosmolares conducen a un aumento de la PIC, a acidosis metabólica y a insuiciencia renal aguda; mantener la normovolemia con albúmina, cristaloides o sangre; y si el manitol falla o la osmolaridad sérica asciende 25 mOsm.L-1 por encima del nivel normal, usar furosemida ${ }^{10}$.

\section{Hiperventilación}

Mantener la $\mathrm{pCO}_{2}$ entre $30-35 \mathrm{~mm} \mathrm{Hg}$.

\section{Coma farmacológico}

Emplear pentobarbital sódico en dosis de carga de $10 \mathrm{mg} / \mathrm{Kg}$ en 30 minutos y $5 \mathrm{mg} / \mathrm{Kg}$ cada hora durante 3 horas, seguido de una dosis de mantenimiento de $1 \mathrm{mg} / \mathrm{Kg} / \mathrm{h}$. Alternativamente, puede utilizarse una dosis de carga de propofol $(1 \mathrm{mg} / \mathrm{Kg}$ ) durante 10 minutos, seguida de una infusión que comienza con incrementos de $100 \mu \mathrm{g} / \mathrm{Kg} / \mathrm{min}$ cada 5 minutos, hasta que se consiga una supresión de puntas en el EEG en períodos de 4-8 segundos, manteniendo este patrón 5 minutos.

\section{Craniectomía descompresiva}

En pacientes que experimentan HIC mayor de $30 \mathrm{~mm} \mathrm{Hg}$ durante más de 15 minutos, refractaria a las medidas precedentes $^{10}$.

\section{Control metabólico}

Suprimir la acidosis metabólica, los estados hiperglucémicos por diabetes, fiebre, sepsis y crisis comiciales, ya que incrementan el CMRO2 y, por tanto, reducen el estrecho margen que permite disminuir el FSC (entiéndase PPC) y, secundariamente, la PIC. Se recomienda el empleo de hipotermia moderada (35-35,5C) y la aplicación de métodos invasivos para el control metabólico. Como terapia experimental, podría ser aconsejable añadir vitamina E (400 U/ día por vía intravenosa o 1000 U cada 8 horas por sonda nasogástrica) y vitamina C (1 g en $100 \mathrm{ml}$ de suero con dextrosa al $5 \%$ cada 8 horas $)^{10}$.

Recibido: 03 de mayo de 2017 Aceptado: 10 de junio de 2017 


\section{Referencias}

1. Gamal Hamdan Suleiman MD. Trauma Craneoencefálico Severo: Parte I.

2. Ministerio de Salud de Colombia - Colciencias - Fundación MEDITECH. Guías de Práctica Clínica para el Diagnóstico y Tratamiento del Trauma Craneoencefálico en Colombia. Sitio Web. (Visitado Sept. 15 - 2013. Disponible en: http://andresrubian6. wix.com/guiastcemeditech\#!)

3. Rubiano AM, Yepes R. Neurotrauma y Neurointensivismo. 1를 Editorial Distribuna. 2009.

4. Alted E, Bermejo S, Chico M. Actualizaciones en el manejo del traumatismo craneoencefálico grave. UCI Trauma. Servicio de Medicina Intensiva. Hospital Universitario 12 de Otubre. Madrid, España.

5. Santacruz L, Herrera A. Universidad de Salamandra (2014); Trauma - Abordaje inicial de los servicios de Urgencias - Cap. 12 Trauma Craneoencefálico (231-255).

6. Machio PS, Previvliano IJ, Goldini CE, Murillo-Cabezas F. Traumatismo Craneoencefálico en la Ciudad de Buenos Aires: Estudio epidemiológico prospectivo de base poblacional. Neurocirugía 2006; 17.

7. Instituto Nacional de Medicina Legal. Forensis 2014. Bogotá, Colombia, 2014.

8. Pradilla G, Vesga BE, et al. Estudio neuroepidemiológico nacional (EPINEURO) colombiano. Rev Panam Salud Publica/Pan Am J Public Health 14(2), 2003.

9. Guzmán F., Colombia Médica - Universidad de Valle. Fisiopatología del Trauma Craneoencefálico (Vol. 39, No. 3 Supl 3 (2008). Sitio Web (visitado Ener. 27; 2017.

10. Bárcena-Orbe CA, Rodríguez-Arias B, Rivero-Martín JM, et al. Revisión del traumatismo craneoencefálico. Neurocirugía 2006; 17: 495518.

11. Pérez G. Urgencias Médico Quirúrgico - Escalas de Valor del Paciente politraumatizado. 2015.

12. Ducker S. Effect of intracranial pressure monitoring and aggressive treatment on mortality in severe head injury. J Neurosurg. 1982; 56: 498-503.

13. Stein SC, Ross SE. Moderate head injury; a guide of initial management. J Neurosurg. 1992; 77: 562-564.

14. Taneda M, Kataoka K. Traumatic subarachnoid hemorrhage as a predictable indicator of delayed ischemic symptoms. J Neurosurg. 1996; 84: 762-768.

15. Bullock R, Chestnut R, Clifton G, et al. Guidelines for the management of severe head injury. Brain Trauma Foundation. Eur J Emerg Med. 1996; 3: 109-127.

16. Unterberg A, Kienning KL. Multimodal monitoring in patients with head injury. Evaluations of the effects of treatment on cerebral oxygenation. J Trauma. 1997; 42 (Suppl 5): 32-37.

17. Miller JD, Butterworth JF, Gudeman SK. Further experience in the management of severe head injury. J Neurosurg. 1996.

18. Paolin A, Nardin L, Gaetani P. Rodríguez y Baena R, Pansarasa O, Marzatico F. Oxidative damage after severe head injury and its relationship to neurological outcome. Neurosurgery 2002; 51: 949-955.

19. Moscote Salazar LR. Trauma Craneoencefálico - Enfoque Básico en Urgencias 2015.

20. Foulkes MA, Eisenberg HM, Jane JA, Marmarou A, Marshall LF. The traumatic coma data bank: design, methods, and baseline characteristics. J Neurosurg (Suppl) 1991; M75: S8-S13.

21. Rubiano A, Charry J, Garzón E. Trauma Craneoencefálico Grave - Green Book (2013); 11: 110-128.

22. North B, Reilly P. Raised intracranial pressure. A clinical guide. Oxford, Heinemann Medical Books, 1990.

23. Teasdale GM, Graham DI. Craniocerebral trauma: protection and retrieval of the neuronal population after injury.

24. Smith DH, Casey K, McIntosh TK. Pharmacologic therapy for traumatic brain injury: experimental approaches. New Horizons 1995; 3 : 562-572.

\section{Correspondencia a:}

José Daniel Charry

Médico Investigador Fundación Universitaria Navarra, UNINAVARRA, Neiva, Colombia.

danielcharry06@gmail.com 